Case Report

\title{
Neurofibromatosis Type I and Stromal Tumor with a Multiple Digestive Localization
}

\author{
Amina Chaka, Farouk Ennaceur (D), Mohamed Amine Tormen, Ibtissem Korbi, \\ Faouzi Noomen, and Khadija Zouari
}

\begin{abstract}
Department of General and Digestive Surgery at the University Hospital Fattouma Bourguiba of Monastir, University of Monastir, Tunisia
\end{abstract}

Correspondence should be addressed to Farouk Ennaceur; enn.farouk92@gmail.com

Received 18 May 2021; Accepted 2 August 2021; Published 21 October 2021

Academic Editor: Mario Ganau

Copyright (C) 2021 Amina Chaka et al. This is an open access article distributed under the Creative Commons Attribution License, which permits unrestricted use, distribution, and reproduction in any medium, provided the original work is properly cited.

\begin{abstract}
Neurofibromatosis type I (NF1) is also known as von Recklinghausen disease. It is a genetic disorder that affects the growth and development of nerve cell tissue, which is characterized by a multisystem disorder and an increased risk for cancer. The incidence of gastroduodenal stromal tumor during Recklinghausen disease can reach $35 \%$ in autopsies and 5\% in clinical cases. In our case, the diagnosis of neurofibromatosis type I was made in a middle-aged women initially diagnosed with a pancreaticoduodenal tumor.
\end{abstract}

\section{Introduction}

Neurofibromatosis type I is also known as von Recklinghausen disease. It is a genetic disorder that affects the growth and development of nerve cell tissue, which is characterized by a multisystem disorder and an increased risk for cancer.

The incidence of gastroduodenal stromal tumor during Recklinghausen disease can reach to $35 \%$ in autopsies and to $5 \%$ in clinical cases.

In our case, the diagnosis of neurofibromatosis type I was made in a middle-aged woman initially diagnosed with a pancreaticoduodenal tumor.

\section{Case Report}

It is a 61-year-old woman, having a regular checkup for rheumatoid arthritis in the rheumatology department. She was admitted in our department for epigastralgia as well as pain on the right hypochondrium, evolving for two days without fever.

The general examination of this patient did not show any anomalies. The patient was in a good general condition with a perfectly supple abdomen with no fever. However, we observed brownish skin lesions, also known as café au lait spots or coffee with milk spots that consists in hyperpigmented lesions that may vary in color from light brown to dark brown (Figure 1). Also, the patient has nodular cutaneous lesions with millimeter size and dots on the skin especially on the anterior surface of the thigh. These lesions correspond to neurofibromas (Figure 2).

Abdominal ultrasound showed a heterogeneous retroperitoneal nodular lesion, vascularized with the presence of multiple partitions to the color Doppler (Figure 3).

The level of tumor markers (CA19-9 and CEA) was normal. The abdominal CT scan showed a $5 \mathrm{~cm}$ juxta duodenal mass, suggesting a stromal tumor. It does not show any reverberation or signs of locoregional extension (Figure 4).

We completed our examination by performing a gastrointestinal endoscopy, which revealed a large, easily identifiable round and heterogeneous lesion which was calcified and vascularized, with collateral circulation growth and a tissue mass component of the pancreas measuring $5 \mathrm{~cm}$. Immunohistochemistry analysis showed an elevated level of vimentin and alpha-fetoprotein (AFP) and the absence of keratin and Synaptophysin.

As a result, this patient was diagnosed with solid pseudopapillary tumor of the pancreatic cephalic area. The tumor 


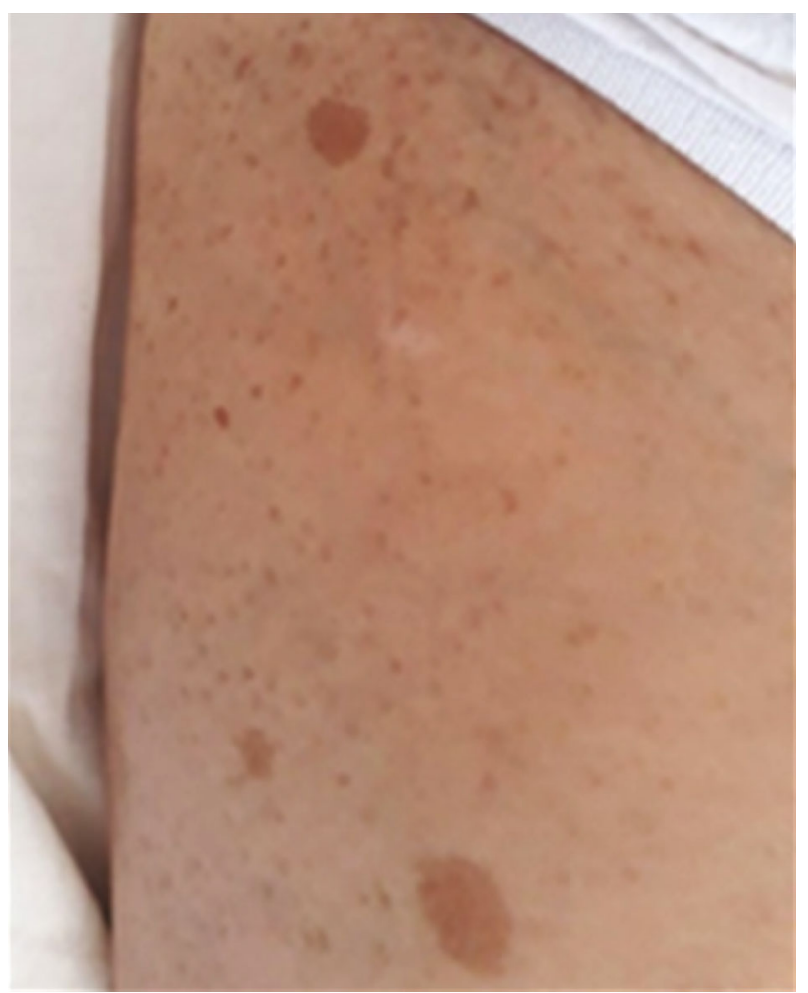

Figure 1: Café au lait spots or coffee with milk spots; it consists in hyperpigmented lesions that may vary in color from light brown to dark brown.

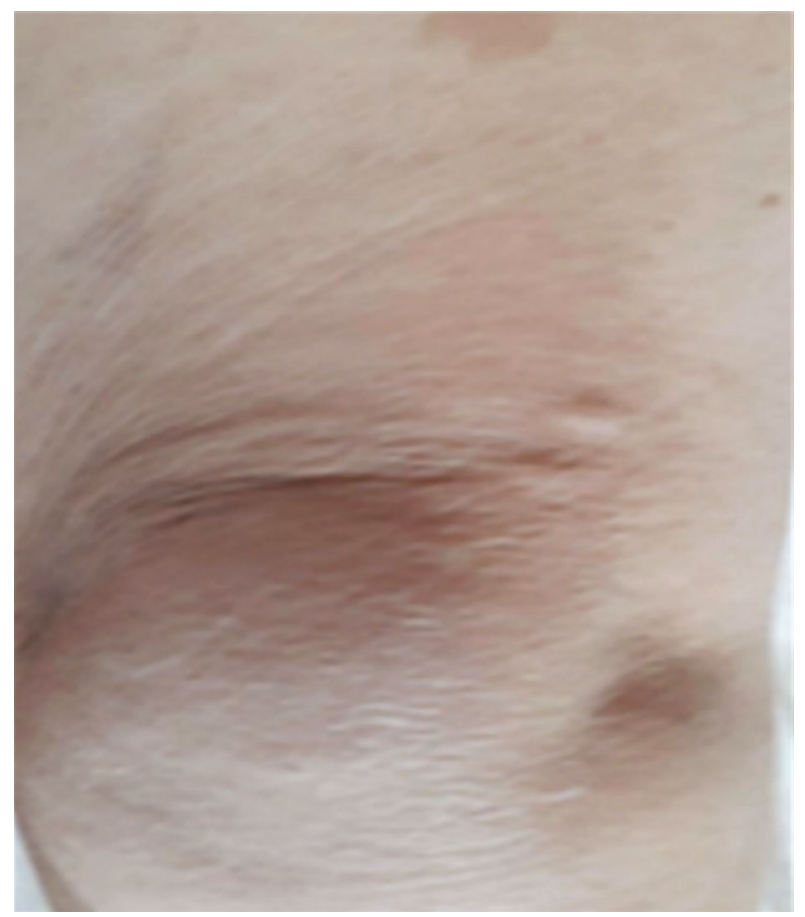

FIgURE 2: Neurofibromas on the anterior thigh. extension assessment was negative, and an operability test was carried out. As a conclusion, the patient undertook a pancreaticoduodenectomy, and intraoperative exploration revealed a tumor measuring $7 \mathrm{~cm}$, with a significant collateral venous circulation, located at D1 and independent of the pancreas (Figure 5). The exploration confirmed the existence of multiple small exophytic lesions, located at the surface of the small bowel. These lesions look like fibroids; biopsy was performed.

In addition, biopsy was carried out on multiple flat millimeter lesions located at the stomach surface. Moreover, no hepatic or peritoneal metastasis was found, as well as no lymphatic metastasis. Excision of the tumor was carried out with extemporaneous examination with negative surgical margins.

We proceeded a surgical resection of a part of the small intestine, in which exophytic nodules were located at. Histopathological examination revealed a stromal tumor in the stomach, small intestine, and duodenum. The postoperative course was simple with a dietary recovery at day 5 . The patient was discharged 7 days postoperatively.

\section{Discussion}

Neurofibromatosis type I is also known as Von Recklinghausen disease after the German researcher, Friedrich Daniel von Recklinghausen, first described it in 1881 as a genetic neurodevelopmental disease, characterized by multisystemic disorders, including an increased risk of cognitive impairment ranging between 50 and $70 \%$, and susceptibility to cancer [1]. Considered one of the most common genetic diseases, it has an estimated incidence of $1 / 3000$ and a prevalence of $1 / 5000$. Almost half of the people suffering from this disease were affected because of novo mutation [2]. Besides gastrointestinal manifestations and neuroendocrine tumors in patients with NF1, they share similarities with other conditions such as multiple endocrine neoplasia type 2B (MEN2B), Cowden syndrome (PTEN mutation), ganglioneuromas (GNs), and Proteus syndrome (PT); usually, those conditions share a genetic background of somatic mosaicisms implicating the PI3K-AKT-mTOR pathway activation which is now amenable to chemotherapy strategies [3].

The diagnostic criteria of neurofibromatosis type I were established in 1988, and it is confirmed when at least two of the following signs are found [4] (Table 1).

There is no accurate treatment to cure this disease. Recklinghausen disease occurs at middle age, usually much later than skin lesions, and can be divided into four entities [5]: (a) lesions of the intrinsic digestive nervous system and its supporting tissues, (b) stromal tumors, (c) duodenal neuroendocrine tumors or of the periampullary region, and (d) various tumors that do not belong to any of the previous tumors. It is noteworthy that the digestive system is affected by this disease in 12 to $60 \%$ of the cases.

As they are located next to the concerned digestive, the liver and the pancreas can also be affected. The incidence of stromal tumors of the digestive tract during Recklinghausen disease was approximatively up to $35 \%$ in the autopsy 

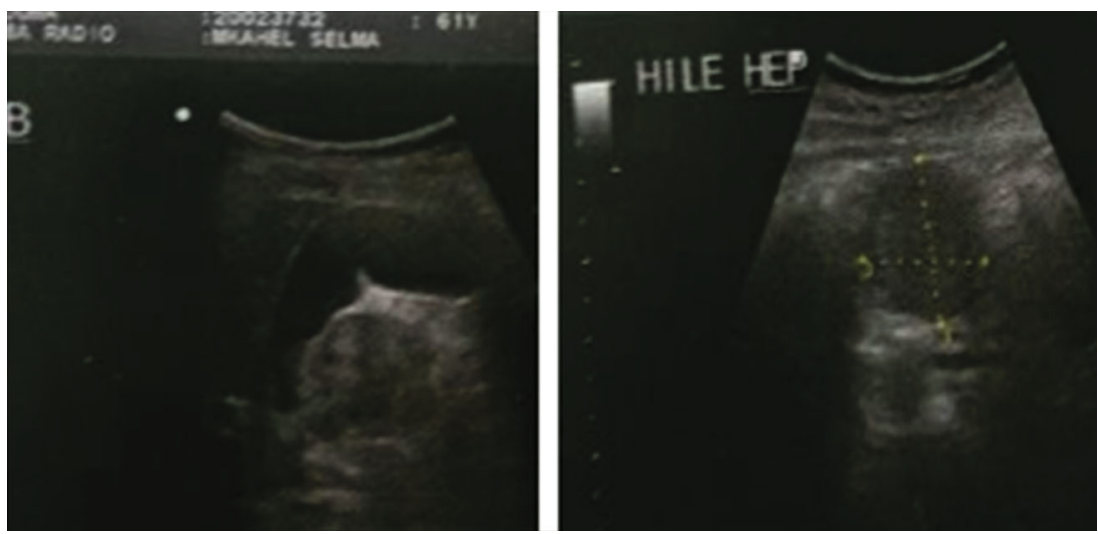

FIgURE 3: Abdominal ultrasound showing heterogeneous retroperitoneal nodular lesion, vascularized using color Doppler in presence of multiple partitions.

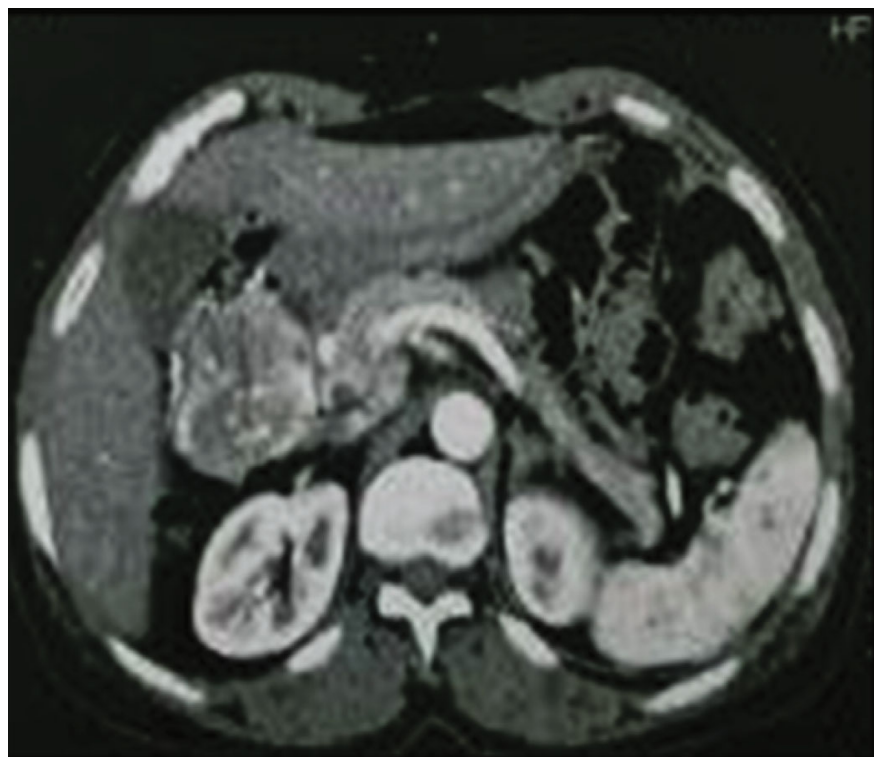

Figure 4: Abdominal CT enhanced scan shows a $5 \mathrm{~cm}$ exophytic duodenum mass, revealing a stromal tumor.
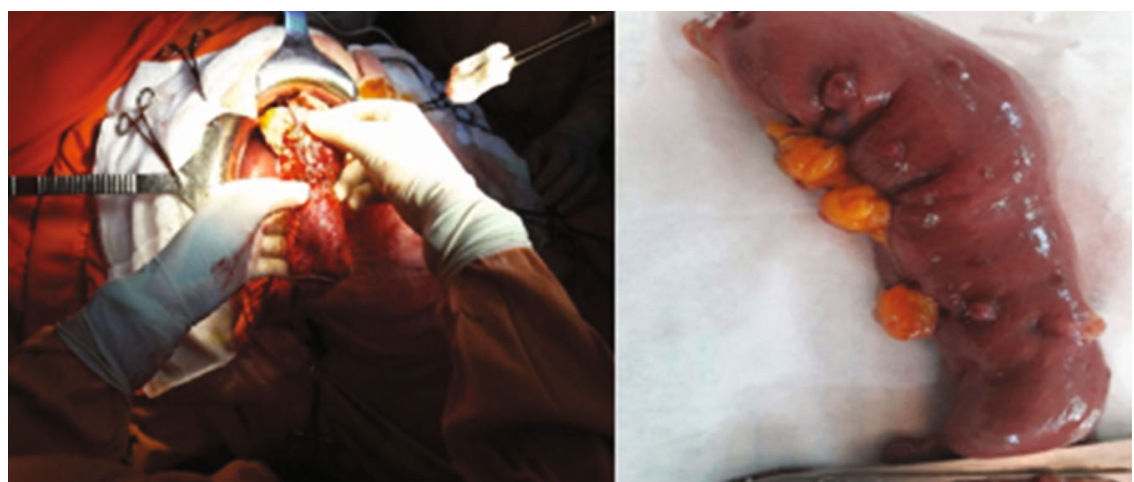

Figure 5: A tumor located $7 \mathrm{~cm}$ from the major axis, located at D1 with exophytic lesions, of centimeter size, located at the surface of the small bowel, resembling fibroids. 
TABLE 1: Clinical diagnostic criteria for neurofibromatosis type I.

Diagnostic criteria of NF1
Two of the following signs
Six colored spots or more, measuring at least $5 \mathrm{~mm}$ before puberty
and $15 \mathrm{~mm}$ after puberty
Lentigines in the axillary or inguinal region
Optic nerve glioma
Two neurofibromas of any type or plexiform fibroid
Two or more s
Bone abnormalities such as a dysplastic sphenoid bone or very fine
long bone cortex
First-degree relative suffering from this disease

cases and only $5 \%$ in the clinical cases. Therefore, these tumors that can be either benign or malignant have a certain latency [6]. In case the patient suffers from multiple stromal tumors, it is important to look for Recklinghausen disease in both the patient and his family even in the absence of this disease's stigma. When dealing with Recklinghausen disease, one should always keep in mind the risk of a malignant tumor since it is four times higher in this disease than in the general population [7, 8]. It has been shown in the literature that histological diagnosis is not enough to find out if the tumor is malign or benign, leading to prognostic uncertainties. However, if the mitotic index is greater than five mitoses for 50 high-magnification fields and the size is greater than $5 \mathrm{~cm}$, it is very much likely a malignant tumor [6]. The diagnostic circumstances of gastrointestinal stromal tumors are variable, including adventitious discovery, pain, mass syndrome, anemia, hem peritoneum, and especially gastrointestinal hemorrhage, which is the most frequent symptom.

Surgery remains the most used treatment for this disease. The only limitation facing this treatment is the multiple localizations of the gastrointestinal tumors, constraining the excision to symptomatic or complicated localizations [9]. Even patients with incomplete features to justify a diagnosis for NF1 still share common needs about the surgical management of some rare forms of tumors [10].

In all stages combined, survival after resection of malignant gastrointestinal stromal tumors is $35 \%$ at 5 years [11]. For an isolated primary tumor, survival is $50-56 \%$ at 5 years and $35-43 \%$ at 10 years with a more favorable prognosis for the stomach than for the small intestine $[11,12]$. Surgery is the only effective treatment for an isolated tumor, while chemotherapy is deemed ineffective in the case of metastases $[4,13]$.

\section{Conclusion}

In cases with multiple stromal tumors, it is important that physicians and other caretakers be more aware of the potential association with Recklinghausen disease even in the absence of this disease's stigma knowing that they are at higher risk of a malignancy, since it is four times higher in this disease than in the general population.

\section{Consent}

We had the written consent of the patient.

\section{Conflicts of Interest}

We, the authors, declare that there is no conflict of interest regarding the publication of this article.

\section{Authors' Contributions}

All the authors have read and agreed to the final manuscript.

\section{References}

[1] A. Hirbe and D. Gutmann, "Neurofibromatosis type 1: a multidisciplinary approach to care," The Lancet Neurology, vol. 13, no. 8, pp. 834-843, 2014.

[2] K. Jett and J. M. Friedman, "Clinical and genetic aspects of neurofibromatosis 1," Genetics in Medicine, vol. 12, no. 1, pp. 1-11, 2010.

[3] J. M. Nicácio, J. C. Rodrigues, M. H. Galles, I. V. Faquini, C. A. de Brito Pereira, and M. Ganau, "Cervical intramedullary schwannoma: a case report and review of the literature," Rare Tumors., vol. 1, no. 2, article e44, pp. 137-140, 2009.

[4] N. E. Julian, N. E. Edwards, S. DeCrane, and C. Hingtgen, "Neurofibromatosis 1: Diagnosis and Management," The Journal for Nurse Practitioners, vol. 10, no. 1, pp. 30-35, 2014.

[5] T. Rosenbaum and K. Wimmer, "Neurofibromatosis type 1 (NF1) and associated tumors," Klinische Pädiatrie, vol. 226, no. 6-7, pp. 309-315, 2014.

[6] C. E. Fluller and G. T. Williams, "Gastrointestinal manifestations of type 1 neurofibromatosis (von Recklinghausen's disease)," Histopathology, vol. 19, no. 1, pp. 1-12, 1991.

[7] R. Ricci, M. Martini, T. Cenci et al., "Divergent gastrointestinal stromal tumors in syndromic settings," Cancer Genetics, vol. 209, no. 7-8, pp. 354-358, 2016.

[8] M. E. T. Zoller, B. Rembeck, A. Oden, M. Samuelsson, and L. Angervall, "Malignant and benign tumors in patients with neurofibromatosis type 1 in a defined Swedish population," Cancer, vol. 79, no. 11, pp. 2125-2131, 1997.

[9] V. P. Grignol and P. M. Termuhlen, "Gastrointestinal stromal tumor surgery and adjuvant therapy," Surgical Clinics of North America, vol. 91, no. 5, pp. 1079-1087, 2011.

[10] Y. Duan, J. Haybaeck, and Z. Yang, "Therapeutic potential of $\mathrm{PI} 3 \mathrm{~K} / \mathrm{AKT} / \mathrm{mTOR}$ pathway in gastrointestinal stromal tumors: rationale and progress," Cancers, vol. 12, no. 10, p. 2972, 2020.

[11] K. P. Boyd, B. R. Korf, and A. Theos, "Neurofibromatosis type 1," Journal of the American Academy of Dermatology, vol. 61, no. 1, pp. 1-14, 2009.

[12] M. Beltran, K. Cruces, C. Barria, and G. Verdugo, "Multiple Gastrointestinal stromal tumors of the ileum and neurofibromatosis type 1," Journal of Gastrointestinal Surgery, vol. 10, no. 2, pp. 297-301, 2006.

[13] S. Y. Wang and T. J. Tan, "A case of a patient with neurofibromatosis type I presenting with abdominal pain," Annals of the Academy of Medicine, Singapore, vol. 45, no. 11, pp. 524-526, 2016. 